\title{
Functional analysis of the nuclear localization signal of the POU transcription factor Skn-1a in epidermal keratinocytes
}

\author{
RYUTA MORITSUGU, KATSUTO TAMAI, HAJIME NAKANO, TAKAYUKI AIZU, \\ KOJI NAKAJIMA, TAKEHIRO YAMAZAKI and DAISUKE SAWAMURA \\ Department of Dermatology, Hirosaki University Graduate School of Medicine, \\ Hirosaki, Aomori 036-8562, Japan
}

Received February 18, 2014; Accepted May 22, 2014

DOI: $10.3892 /$ ijmm.2014.1803

\begin{abstract}
POU domain proteins are a family of critical regulators of development and differentiation due to their transcriptional activity in the nucleus. Skn-1a, a member of the POU domain protein family, appears to be expressed predominantly in epidermal keratinocytes and is thought to play a critical role in keratinocyte differentiation and proliferation. In this study, we examined the mechanisms involved in the nuclear localization of Skn-1a. We transiently expressed enhanced green fluorescent protein (EGFP) reporter constructs encoding EGFP fusions with Skn-1a deletion and mutation proteins in normal human epidermal keratinocytes (NHEKs). The experiments clearly demonstrated that Skn-1a contained a functional nuclear localization signal (NLS) domain, and that the smallest domain necessary for Skn-1a nuclear transport was the GRKRKKR sequence located within amino acids 279-285. Previous studies have shown that the phosphorylation of specific amino acids neighboring the NLS may regulate nuclear transport and that the amino acid residues threonine (Thr) and serine (Ser) have the potential to undergo phosphorylation. We examined whether the amino acids Thr286 and Ser287, which reside adjacent to the NLS at the carboxy-terminal side, play a role in Skn-1a nuclear localization. For this purpose, we generated three EGFP-Skn-1a mutation constructs, in which Thr286, Ser287, or both Thr286 and Ser287 residues were replaced with alanine, respectively. The results showed that the Thr286 and Ser287 residues were involved in the regulation of nuclear localization as well as
\end{abstract}

Correspondence to: Professor Daisuke Sawamura, Department of Dermatology, Hirosaki University Graduate School of Medicine, 5 Zaifu-cho, Hirosaki, Aomori 036-8562, Japan

E-mail: smartdai14@yahoo.co.jp

Abbreviations: EGFP, enhanced green fluorescent protein; NHEK, normal human epidermal keratinocyte; NLS, nuclear localization signal; PMA, phorbol 12-myristate 13-acetate

Key words: differentiation, skin, phorbol 12-myristate 13-acetate, nuclear pore complex epidermal differentiation. These results suggested that the epidermal differentiation signaling pathway, involving kinase and phosphatase activation, may regulate the NLS activity of Skn-1a in keratinocytes. Collectively, these data contribute to understanding the mechanisms of nuclear translocation of POU domain proteins and epidermal differentiation.

\section{Introduction}

The epidermis is a stratified squamous epithelium in which viable cells move outwardly from the basal layer to become terminally differentiated keratinocytes that eventually constitute the stratum corneum. Keratinocyte proliferation and differentiation are closely regulated by cellular transcription factors, including activator protein-1 family proteins (1), nuclear factor- $\kappa \mathrm{B}$ family proteins (2), cytidine-cytidine-adenosineadenosine-thymidine/enhancer-binding proteins (3), p53-related proteins (4), and POU transcription factors (5). POU transcription factors are characterized by a bipartite POU domain in which a homeodomain is connected by a short linker region to an N-terminally located POU-specific domain (6). The POU-specific and POU homeodomain are DNA-binding domains of the helix-turn-helix type.

Skn-1a, a member of the POU domain transcription factor family, appears to be expressed predominantly in the epidermal keratinocytes and is thought to play a critical role in keratinocyte differentiation and proliferation (7-9). Skn-1a transactivates the expression of the genes encoding K10 and SPRP2A, which are expressed during keratinocyte differentiation, suggesting that Skn-1a promotes keratinocyte differentiation. Furthermore, we have previously reported that the mRNA expression of Skn-1a increases in cultured normal human keratinocytes subsequent to calcium-induced differentiation $(9,10)$.

Similar to POU domain proteins characterized thus far (2), Skn-1a exerts its function in the nucleus. Nuclear proteins enter this cellular compartment via the nuclear pore complex after being synthesized in the cytoplasm, and usually, they are actively transported through the nuclear pore (11). Such active transportation requires energy, transport receptors, and an endogenous nuclear localization signal (NLS) within the cargo protein.

NLSs have been identified in a variety of nuclear proteins ranging in size from $<100$ to $>1,000$ amino acids, 
including polymerases, kinases, phosphatases, transcription factors, histones, tumor suppressor molecules, and various viral proteins. In the present study, we characterized the NLS of Skn-1a, which was shown to be localized within its DNA-binding domain as a motif highly conserved among the POU domain proteins. Its identification enhances our understanding of the evolution of POU domain proteins and the mechanisms involved in regulating the access of POU domain proteins to the nucleus.

\section{Materials and methods}

Enhanced green fluorescent protein (EGFP)-Skn-la deletion and mutation reporter constructs. The EGFP-Skn-1a plasmid was constructed as described below (7). First the Skn-1a coding region, which contains the canonical NLS consensus sequence, GRKRKKR (aa279-285), was amplified by performing PCR and subcloned into the C-terminus of pEGFP (Clontech Laboratories, Palo Alto, CA, USA). The primers were used for PCR, BsrGI-207; 5'-GGGTGTACA AGTTCACACAGGGAGATGGGCTGGCGA-3' and NotI-295; 5'-TATGCGGCCGCAGTCAGGCGGATGTTG GTCTC-3' for the 207-295 fragment, BsrGI-207 and NotI-287; 5'-GGGGCGGCCGCTTTAGCTGGTCCGTTTCTTTCT CTTCCTACCAAA-3' for the 207-287 fragment, $B s r$ GI-258; 5'-GGGTGTACCCTCTCCGTCAGA CCCCTCAGTG-3' and NotI-287 for the 258-287 fragment, BsrGI-269; 5'-GGGTGT ACACCTCCTACCCCAGCCTCAGTGAA-3' and NotI-287 for the 269-287 fragment. The 273-287, 279-287 and 279-285 fragments were directly employed as the following linkers: 5'-GTACAGGCTCAGTGAAGTATTTGCTAGGAAGAG AAAGAAACGGACCAGCGC-3' and 5'-GGCCGCGCT GGTCCGTTTCTTTCTCTTCGTACCAAATACTTCACT GAGCCT-3' for the 273-287 fragment, 5'-GTACAC CGGTAGGAAGAGAAAGAAACGGACCAGCGC-3' and 5'-GGCCGCGCTGGTCCGTTTCTTTCTCTTCCTAC CGGT-3' for the 279-287 fragment, 5'-GTACACCGGTAG GAAGAGAAAGAAACGGACCAGCGC-3' and 5'-GGCCG CCCGTTTCTTTCTCTTCCTACCGGT-3' for the 279-285 fragment. The EGFP-Skn-1a deletion constructs pEGFPSkn-1a 207-295, pEGFP-Skn-1a 207-287, pEGFP-Skn-1a 258-287, pEGFP- Skn-1a 269-287, pEGFP-Skn-1a 273-287, pEGFP-Skn-1a 279-287 and pEGFP-Skn-1a 279-285 were generated by cloning the corresponding segments into pEGFP as $B s r$ GI-NotI fragments. We also constructed the mutation constructs pEGFP-Skn-1a 279-285m in which both Arg282 and Lys 283 were replaced by alanines using the following linkers: 5'-GTACACCGGTAGGAAGGCTGCTAAACG GACCAGCGC-3' and 5'-GGCCGCCCGTTTAGCAGCCTT CCTACCGGT-3'.

Plasmids for the EGFP-Skn-1a mutation reporter constructs EGFP-Skn-1a 207-287m1 in which threonine (Thr) 286 was replaced with alanine, EGFP-Skn-1a 207-287m2 in which serine (Ser) 287 was replaced with alanine, and EGFPSkn-1a 207-287m3 in which both Thr286 and Ser287 were replaced with alanine, were constructed from pEGFP1Skn-1a $258-287$ by PCR with mutagenetic primers (forward for all, BsrGI-207; 5'-GGGTGTACAAGTTCACACAGGGAG ATGGGCTGGCGA-3'; reverse, 5'-GGGGCGGCCGCTT TAGCCGGTCCGTTTCTTTCTCTTCCTACCAAA-3' for m1, 5'-GGGGCGGCCGCTTTAGCTGCCCCGTTTCTTTC TCTTCCTACCAAA-3' for $\mathrm{m} 2$, and 5'-GGGGCGGCCG CTTTAGCCGCCCCGTTTCTTTCTCTTCCTACCAAA-3' for $\mathrm{m} 3$ ).

Cell culture. Normal human epidermal keratinocytes (NHEKs) from neonatal foreskin were obtained commercially (Clonetics, San Diego, CA, USA). Cultures were grown in a $60-\mathrm{mm}$ culture dish in keratinocyte growth medium containing human recombinant epidermal growth factor $(0.1 \mathrm{ng} / \mathrm{ml})$, insulin $(5 \mathrm{ng} / \mathrm{ml})$, hydrocortisone $(0.5 \mathrm{ng} / \mathrm{ml})$, gentamicin $(50 \mathrm{ng} / \mathrm{ml})$, and amphotericin-B (50 ng/ml). Phorbol 12-myristate 13-acetate (PMA, Sigma-Aldrich, St. Louis, MO, USA) was used to induce keratinocyte differentiation.

Transfection and microscopy. The plasmid constructs described above were used in transient transfection studies in cultured NHEKs $(9,10)$. After the cells had grown to $70 \%$ confluence, they were transfected with $5 \mathrm{ng}$ of the EGFP-Skn-1a expression vector using the DOTAP Liposomal Transfection Reagent (Roche Applied Science, Indianapolis, NJ, USA). The pEGFP plasmid was used as the control. The cells expressing EGFP were analyzed by fluorescence microscopy of 10 randomly selected fields. The cells were considered positive when the nuclear fluorescence signal was clearly stronger that the cytoplasmic fluorescence signal. The total number of cells expressing EGFP (A) and the number of the positive cells in which EGFP was localized in the nucleus (B) were measured, and the rate of $\mathrm{B} / \mathrm{A}$ was calculated. The rate of nuclear translocation of each construct was expressed as a percentage relative to that of EGFP-Skn-1a 279-285 which contained the most minimum component of the DNA fragment.

Western blot analysis. Western blot analysis was performed following a routine method $(9,10)$. Briefly, the transfected cells were lysed by the sample buffer, electrophoresed on $15 \%$ polyacrylamide gels, and then transferred onto a polyvinylidene difluoride membrane. The membranes were incubated with monoclonal anti-GFP antibodies (Roche Diagnostic Corp., Indianapolis, IN, USA), followed by incubation with a horseradish peroxidase-labelled secondary anti-mouse antibody. Immunocomplexes were visualized using visualized using the Image Lab System (Bio-Rad, Hercules, CA, USA). Anti- $\beta$-actin antibodies were used as a control.

Statistical analysis. The Student's t-test was used to determine the level of significance of differences in sample means. $P<0.01$ was considered significant. Data were shown as mean \pm SD.

\section{Results}

Nuclear translocation of the EGFP-Skn-la deletion proteins. To identify the regions of the $S k n-1$ a protein required for its transport into the nucleus, we generated reporter constructs for Skn-la deletion proteins fused to EGFP at their $\mathrm{N}$-termini (Fig. 1). The putative NLS consensus sequence, GRKRKKR is located between amino acids 279 and 285 . We transfected the constructs into NHEKs and analyzed the EGFP fusion protein localization $24 \mathrm{~h}$ later by fluorescence microscopy. Western blot analysis was also performed to 


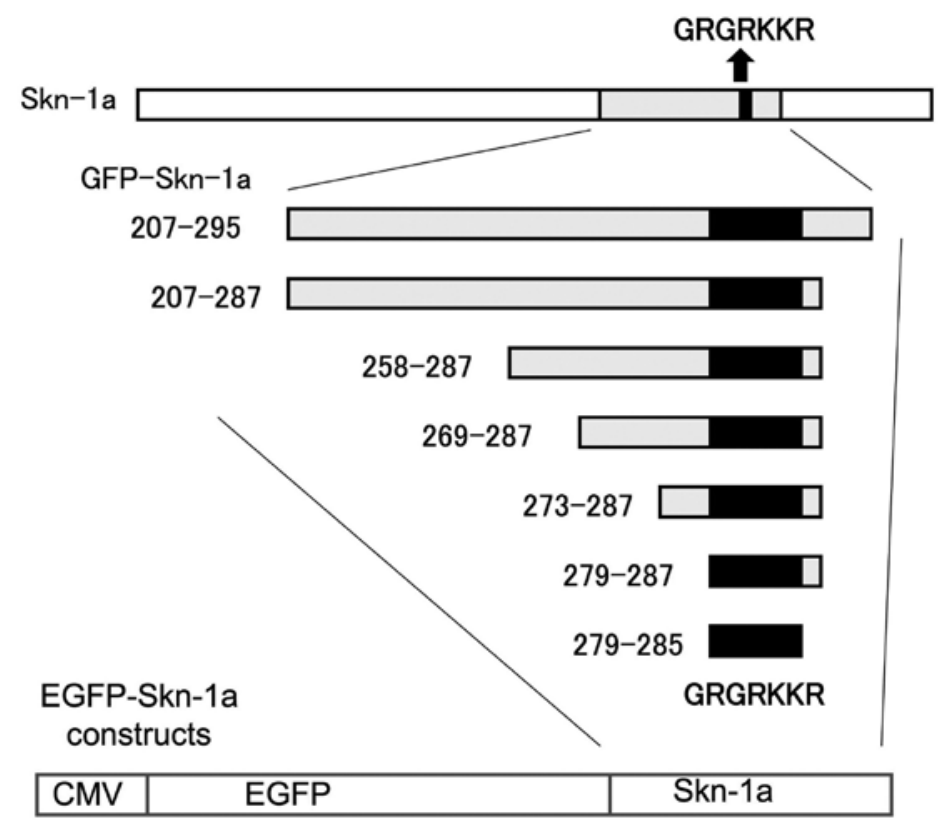

Figure 1. Schematic representation of the enhanced green fluorescent protein (EGFP)-Skn-1a deletion reporter constructs. General structure of the EGFP-Skn-1a constructs. The constructs contain the cytomegalovirus (CMV) promoter and the enhanced green fluorescent protein (EGFP) gene. Skn-1a fragments were fused with the C-terminal amino acid of EGFP. GRGRKKR (aa279-285) is the putative consensus nuclear localization signal (NLS) of Skn-1a.

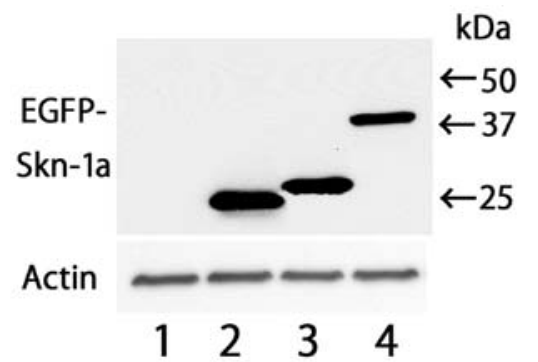

Figure 2. Western blot analysis of the fusion proteins. We transfected the constructs and western blot analysis was performed. Typical experiment was shown in the figure. 1 , no transfection; 2, pEGFP, basic construct; 3 , enhanced green fluorescent protein (EGFP)-Skn-1a 279-285, the shortest EGFP construct; 4, EGFP-Skn-1a 207-287, the longest EGFP construct. Actin, Anti- $\beta$-actin antibodies were used as control.

confirm production of the EGFP fusion protein. The result of western blot analysis revealed that each construct exhibited a band with an estimated size (Fig. 2). Typical positive cells are shown in Fig. 3. The nuclear fluorescence signal was clearly stronger than the cytoplasmic fluorescence signal. Results of the immunofluorescence experiments showed that the rates of nuclear translocation of the fusion proteins from pEGFP-Skn-1a 258-287, 269-287, 273-287, 279-287 and 279-285 were almost identical (Fig. 4). On the other hand, rates for the fusion proteins from the constructs 207-295 and 207-287 were relatively low. The control plasmid pEGFP had little or no ability to enter the nucleus. We also examined the mutation constructs pEGFP-Skn-1a 279-285m in which both Arg282 and Lys 283 were replaced by alanines. The result showed the rate to be identical to that of control pEGFP (data not shown).

Nuclear translocation of the EGFP-Skn-la mutation proteins. The amino acids Thr286 and Ser287 reside adjacent to the NLS on its carboxy-terminal side. We examined their role in Skn-1a nuclear localization by generating three mutation reporter constructs based on the pEGFP-Skn-1a 207-287 construct. Thr286 was replaced with alanine to generate the construct pEGFP-Skn-1a 207-287m1, Ser287 with alanine for the construct pEGFP-Skn-1a 207-287m2, and both Thr286 and Ser287 residues were replaced with alanine for the construct pEGFP-Skn-1a 207-287m3 (Fig. 5). We then compared the nuclear translocation rates of the pEGFP-Skn-1a mutation proteins. The nuclear translocation rates of the pEGFP-Skn-1a207-287m1 andpEGFP-Skn-1a207-287m2 mutation proteins were identical to that of pEGFP-Skn-1a 207-287, whereas the pEGFP-Skn-1a 207-287m3 mutation protein showed a significantly higher rate compared to the other three constructs (Fig. 6).

Effect of PMA treatment on nuclear translocation. PMA has been well characterized as a protein kinase $\mathrm{C}$ activator as well as an inducer of late differentiation marker expression and cornified envelope assembly in vitro. In the subsequent series of experiments we examined the rate of nuclear translocation of the reporter proteins from pEGFP-Skn-1a 207-287, pEGFP-Skn-1a 207-287m1, pEGFP-Skn-1a 207287m2, and pEGFP-Skn-1a $207-287 \mathrm{~m} 3$ in NHEKs treated for $24 \mathrm{~h}$ with $10^{-8}-10^{-6}$ MPMA. The nuclear translocation rates of proteins from pEGFP-Skn-1a 207-287, pEGFP-Skn-1a 207-287m1, and pEGFP-Skn-1a 207-287m2 were enhanced by PMA. However, the rate of the pEGFP-Skn-1a 207-287m3 mutation protein was independent of PMA concentration, and following treatment with $10^{-8} \mathrm{M}$ PMA all four proteins showed essentially the same translocation rate (Fig. 7).

\section{Discussion}

The transport of proteins between the nucleus and cytoplasm occurs primarily through the nuclear pore complex where 

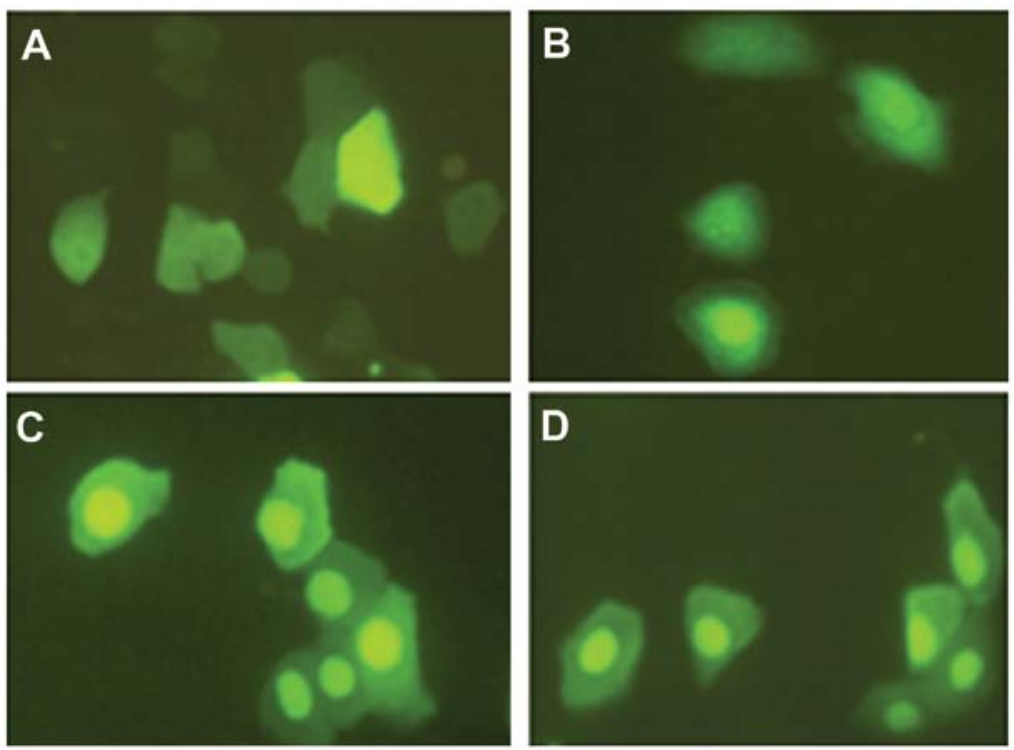

Figure 3. Nuclear translocation of enhanced green fluorescent protein (EGFP)-Skn-1a fusion proteins. We transfected the constructs into normal human epidermal keratinocytes (NHEKs) and analyzed the EGFP fusion protein localization $24 \mathrm{~h}$ later by fluorescence microscopy. Typical positive cells for nuclear translocation are shown. The specimen of pEGFP (A and B) shows no or few positive cells and (C) the majority of cells are positive in the sample of pEGFP-Skn-1a 279-285. (D) Half of the cells are positive in pEGFP-Skn-1a 207-287.

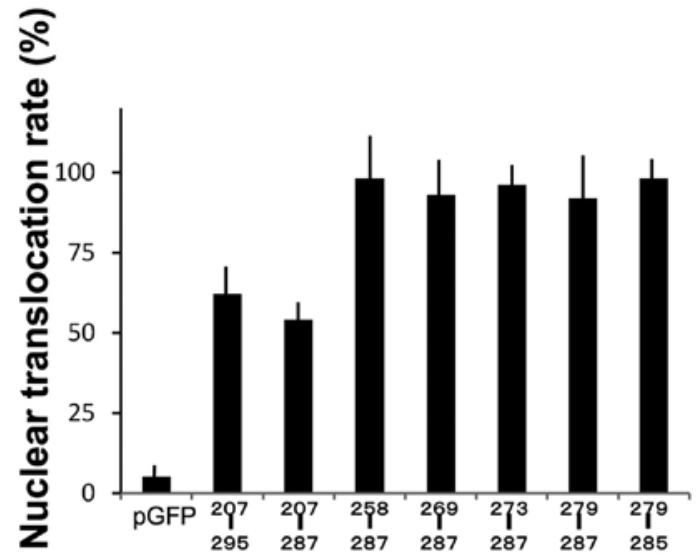

EGFP-Skn-1a constructs

Figure 4. Rates of nuclear translocation of the enhanced green fluorescent protein (EGFP)-Skn-1a deletion proteins in normal human epidermal keratinocytes (NHEKs). Several plasmid constructs, which express Skn-1a deletions as fusion proteins with EGFP were developed and transfected into NHEKs. EGFP-Skn-1a deletion protein localization was analyzed using fluorescence microscopy at $24 \mathrm{~h}$ following transfection. The total number of cells expressing EGFP (A) and the number of the positive cells in which EGFP was localized in the nucleus (B) were measured, and the rate of B/A was calculated. The rate of nuclear translocation of each construct was expressed as a percentage relative to that of EGFP-Skn-1a 279-285, which contained the most minimum component. The indicated values show the mean rate $\pm \mathrm{SD}$.

proteins can enter the nucleus either by diffusion or by signal-mediated transport (11). The structural constraints of the nuclear pore complex established that only proteins with a molecular mass of $<40 \mathrm{kDa}$ are able to enter the nucleus by passive diffusion. However, many proteins are imported by signal-mediated transfer, among them Skn-1a, which is $\sim 48 \mathrm{kDa}$ in size (12). Based on previous observations, we hypothesized that the regulation of nuclear localization of
Skn-1a serves as a molecular switch to control the transcription of various epidermal genes.

The NLStradamus model predicts the sequence GRKRKKR (aa279-285) to be the putative consensus NLS of Skn-1a (13). Therefore, we transiently expressed in keratinocytes a number of EGFP fusion proteins containing the putative NLS with deletions or mutations. The results clearly demonstrate that Skn-1a contains a functional NLS domain, and that the smallest domain necessary for Skn-1a transport is located within amino acids 279-285 (Fig. 2), suggesting that this region of Skn-1a functions as an NLS. Substitution of alanines for both Arg282 and Lys283 eliminated NLS activity of the sequence GRKRKKR (aa279-285). We also found that the deletion proteins from the constructs 207-295 and 207-287 showed a relatively low rate compared with the other shorter constructs, suggesting the presence of a region spanning from 207-287 that potentially inhibits or counteracts the NLS function. It is possible that Ser and Thr residues, prone to phosphorylation, are present in the region 207-258. The computer program NetPhos 2.0 (14), which is utilized to identify potential phospholylation sites, showed that the value of Ser230 was relatively as compared to that of Ser287. Thus, studies targeting Ser230 are to be conducted.

The three POU transcription factors Oct-1, Oct-6, and Skn-1a are expressed in the epidermis. The corresponding NLS regions of these human POU transcription factors Oct-1, Oct-6 and Skn-1a are GLSRRRKKRTSIET, AQGRKRKKRTSIEV, VFGRKRKKRTSIET, respectively (the NSL sequence is underlined) $(7,15,16)$. Notably, all three POU factors contain the identical TSIE amino acid sequence situated at the C-terminus of the NLS. This binding suggests that this area may be important in keratinocyte growth and differentiation. Findings of previous studies have shown that the phosphorylation of particular amino acids neighboring the NLS may regulate NLS activity (17), and that the amino acid residues Thr and Ser are potential candidates for undergoing phosphorylation. 

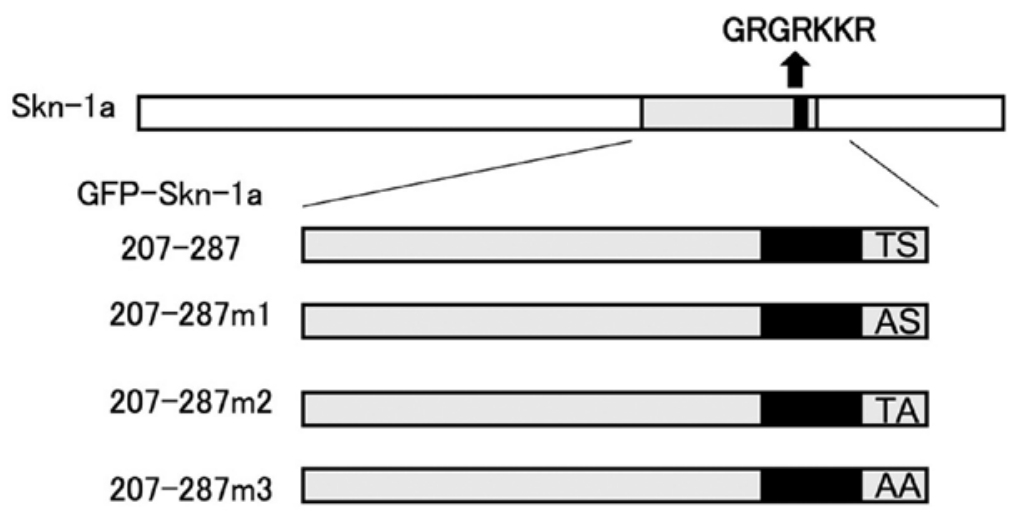

Figure 5. Schematic representation of the enhanced green fluorescent protein (EGFP)-Skn-1a mutation reporter constructs. The fusion protein EGFP-Skn-1a 207-287 contains threonine (Thr) 286 and serine (Ser) 287, which reside adjacent to the nuclear localization signal (NLS) at the carboxy-terminal side. EGFP-Skn-1a 207-287m1 encodes a mutation protein in which Thr286 is replaced with alanine; EGFP-Skn-1a 207-287m2 encodes a mutation protein in which Ser287 is replaced with alanine; EGFP-Skn-1a 207-287m3 encodes a mutation protein in which both Thr286 and Ser287 are replaced with alanines.

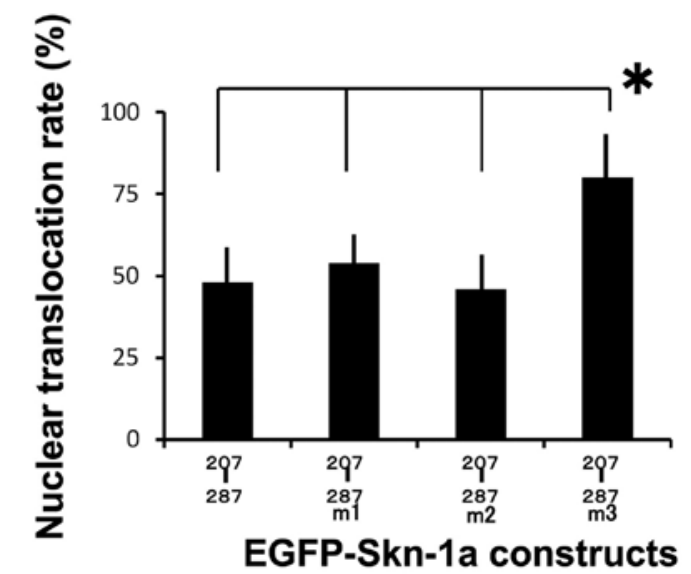

Figure 6. Rates of nuclear translocation of the mutant constructs. We generated enhanced green fluorescent protein (EGFP)-Skn-1a mutation constructs, then transfected them into normal human epidermal keratinocytes (NHEKs), and analyzed the translocation rates. The indicated values are the mean translocation rate \pm SD. Significant differences $\left({ }^{*} \mathrm{p}<0.01\right)$ were found between 207-287 and $\mathrm{m} 3, \mathrm{~m} 1$ and $\mathrm{m} 3$, and $\mathrm{m} 2$ and $\mathrm{m} 3$.

Consequently, we generated three mutation reporter constructs derived from EGFP-Skn-1a 207-287, in which Thr286 was replaced with alanine (EGFP-Skn-1a 207-287m1), Ser287 with alanine (EGFP-Skn-1a 207-287m2), or both Thr286 and Ser287 with alanine (EGFP-Skn-1a 207-287m3) and expressed them in keratinocytes. Results of the present study demonstrate that the nuclear translocation rate of construct $\mathrm{m} 3$ was higher than those of EGFP-Skn-1a 207-287, and constructs $\mathrm{m} 1$ and $\mathrm{m} 2$, suggesting that Thr286 and Ser287 play modulating negative roles in NLS function. We also hypothesize that the nuclear accumulation of Skn-1a correlates with the dephosphorylation of both Thr286 and Ser287.

In the presence of low calcium concentration in the medium, keratinocytes are maintained phenotypically as a basal cell-like population of undifferentiated cells. Under these conditions, the proteins from EGFP-Skn-1a 207-287, EGFP-Skn-1a 207-287m1 and EGFP-Skn-1a 207-287m2 translocate to the nucleus with low efficiency, compared to EGFP-Skn-1a 207-287m3. PMA has been well characterized as an activator of protein kinase $\mathrm{C}$ as well as an

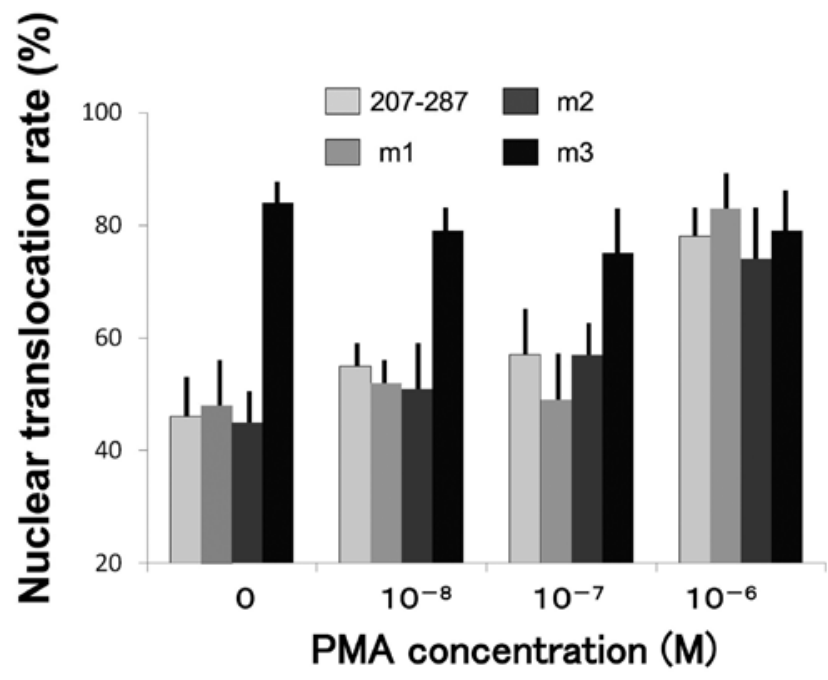

Figure 7. Effect of phorbol 12-myristate 13-acetate (PMA) treatment on the nuclear translocation rate of enhanced green fluorescent protein (EGFP) The extent of nuclear translocation of the deletion and mutation proteins encoded by the EGFP-Skn-1a 207-287, EGFP-Skn-1a 207-287m1, EGFP-Skn-1a 207-287m2 and EGFP-Skn-1a 207-287m3 constructs prior to and following treatment with various concentrations of PMA. Prior to treatment, the calcium concentration in the medium was adjusted to $0.03 \mathrm{mM}$ to maintain a basal cell-like phenotype of undifferentiated cells. The indicated values are the mean translocation rate \pm SD.

inducer of late differentiation marker expression and cornified envelope assembly in vitro. Therefore, we examined the translocation rates of the mutation reporter proteins in differentiated keratinocytes induced by PMA. As a result of PMA treatment, the nuclear translocation rate of proteins from EGFP-Skn-1a 207-287, EGFP-Skn-1a 207-287m1, and EGFP-Skn-1a 207-287m 2 increased. However, the rate of EGFP-Skn-1a 207-287m3 was independent of PMA concentration, and following $10^{-8} \mathrm{M}$ PMA treatment, all four proteins showed essentially the same translocation rate. These data indicate that Thr286 and Ser287 residues play a role in keratinocyte differentiation, suggesting that the epidermal differentiation signaling pathway, involving kinase and phosphatase activation, regulates the NLS activity of Skn-1a in the epidermal keratinocytes. 
Results of the present study suggest that the transcriptional regulation of various epidermal genes provides a differentiation-specific phenotype of the epidermis. At present, the precise mechanism of transcription factor nuclear translocation in keratinocytes remains to be characterized. Further characterization of the transportation mechanisms may facilitate an understanding of signal transduction into the nucleus.

\section{Acknowledgements}

The authors thank Ms. Yuka Toyomaki, Mrs. Yukiko Tamura, Mrs. Yuriko Takagi, and Ms. Nanako Seitoh for their excellent technical assistance. This study was supported in part by Grants-in-Aid from the Ministry of Education, Science, Sports, and Culture of Japan.

\section{References}

1. Eckert RL, Crish JF and Robinson NA: The epidermal keratinocyte as a model for the study of gene regulation and cell differentiation. Physiol Rev 77: 397-424, 1997.

2. Ryan AK and Rosenfeld MG: POU domain family values: flexibility, partnerships, and developmental codes. Genes Dev 11: $1207-1225,1997$

3. Seitz CS, Lin Q, Deng $\mathrm{H}$ and Khavari PA: Alterations in NF-kappaB function in transgenic epithelial tissue demonstrate a growth inhibitory role for NF-kappaB. Proc Natl Acad Sci USA 95: 2307-2312, 1998.

4. Okuyama R, Tagami H and Aiba S: Notch signaling: its role in epidermal homeostasis and in the pathogenesis of skin diseases. J Dermatol Sci 49: 187-194, 2008.

5. Maytin EV, Lin JC, Krishnamurthy $\mathrm{R}$, et al: Keratin 10 gene expression during differentiation of mouse epidermis requires transcription factors $\mathrm{C} / \mathrm{EBP}$ and $\mathrm{AP}-2$. Dev Biol 216 : 164-181, 1999.
6. Herr W, Sturm RA, Clerc RG, et al: The POU domain: a large conserved region in the mammalian pit-1, oct-1, oct- 2 , and Caenorhabditis elegans unc-86 gene products. Genes Dev 2: 1513-1516, 1988.

7. Goldsborough AS, Healy LE, Copeland NG, et al: Cloning, chromosomal localization and expression pattern of the POU domain gene Oct-11. Nucleic Acids Res 21: 127-134, 1993.

8. Andersen B, Weinberg WC, Rennekampff O, et al: Functions of the POU domain genes Skn-1a/i and Tst-1/Oct-6/SCIP in epidermal differentiation. Genes Dev 11: 1873-1884, 1997.

9. Nakajima K, Tamai K, Yamazaki T, et al: Identification of Skn-1n, a splice variant induced by high calcium concentration and specifically expressed in normal human keratinocytes. J Invest Dermatol 128: 1336-1339, 2008.

10. Takemoto H, Tamai K, Akasaka E, et al: Relation between the expression levels of the POU transcription factors Skn-1a and Skn-1n and keratinocyte differentiation. J Dermatol Sci 60: 203-205, 2010

11. McLane LM and Corbett AH: Nuclear localization signals and human disease. IUBMB Life 61: 697-706, 2009.

12. Hildesheim J, Foster RA, Chamberlin ME and Vogel JC: Characterization of the regulatory domains of the human SKN-1a/Epoc-1/Oct-11 POU transcription factor. J Biol Chem 274: 26399-26406, 1999.

13. Nguyen Ba AN, Pogoutse A, Provart N and Moses AM: NLStradamus: a simple hidden Markov model for nuclear localization signal prediction. BMC Bioinformatics 10: 202, 2009.

14. Blom N, Gammeltoft S and Brunak S: Sequence and structure-based prediction of eukaryotic protein phosphorylation sites. J Mol Biol 294: 1351-1362, 1999.

15. Sturm RA, Das G and Herr W: The ubiquitous octamer-binding protein Oct-1 contains a POU domain with a homeo box subdomain. Genes Dev 2: 1582-1599, 1998.

16. Suzuki N, Rohdewohld H, Neuman T, Gruss P and Schöler HR: Oct-6: a POU transcription factor expressed in embryonal stem cells and in the developing brain. EMBO J 9: 3723-3732, 1990.

17. Hübner S, Xiao C and Jans DA: The protein kinase CK2 site (Ser111/112) enhances recognition of the simian virus 40 large T-antigen nuclear localization sequence by importin. J Biol Chem 272: 17191-17195, 1997. 